\title{
The Crab Nebula: observations and a search for UHE $\gamma$-ray flares with LHAASO
}

\section{Lingyu Wang ${ }^{\dagger, *}$, Songzhan Chen, Zhen Cao, Huihai He, Sha Wu, Cong $\mathrm{Li}$, Zhe Li For the LHAASO collaboration}

Institute of High Energy Physics, Chinese Academy of Sciences, Beijing, China TIANFU Cosmic Ray Research Center, Chengdu, China

E-mail: wangly@ihep.ac.cn

The Crab Nebula is a steady radiation source, which has been used as a reference source in very high energy $\gamma$-ray astronomy for calibration and verification of detectors, however the $\gamma$-ray flares around GeV from the Crab Nebula have been observed many times by AGILE and Fermi-LAT. These observations challenge the standard models for particle acceleration in pulsar wind nebula. One square kilometer detector array (KM2A) of the Large High Altitude Air Shower Observatory (LHAASO) is designed to detect $\gamma$-ray sources with high sensitivity at $100 \mathrm{TeV}$. In this work, half of the KM2A detector performance, the observations of the Crab Nebula in the energy range above $10 \mathrm{TeV}$ and the results of searching for $\gamma$-ray flares will be presented using data collected with half of the KM2A and three-quarter KM2A.

37th International Cosmic Ray Conference (ICRC2021),

12-23 July 2021

Berlin, Germany - Online

\footnotetext{
${ }^{*}$ Presenter

$\dagger$ This work is supported by the National Key R\&D Program of China (No. 2018YFA0404201) and the Natural Sciences Foundation of China (No. 11905227, 12022502).
} 


\section{Introduction}

The Crab Nebula is the remnant of a supernova explosion which was recorded in Chinese and Japanese chronicles as a 'guest star' in the year 1054 A.D.[1]. At a distance of $2 \mathrm{kpc}$ from the Earth, the Crab Nebula is the brightest pulsar wind nebula, powered by the ultrarelativistic electronpositron wind from the central neutron star(the Crab Pulsar). It is one of the best-studied objects in astronomy, whose photon emission is steady in almost all wavelength bands of the electromagnetic spectrum from $10^{-5} \mathrm{eV}$ (radio) to $10^{15} \mathrm{eV}$ ( $\gamma$-rays), making it a "standard candle". The spectral energy distribution(SED) is well measured by many ground-based experiments, including both air shower arrays[2-5] and imaging Cherenkov telescopes[6-8]. The observed spectrum around $100 \mathrm{TeV}$ is consistent with a smooth extrapolation of the lower-energy spectrum. As a reference VHE $\gamma$-ray source, the Crab Nebula is often used to check detector performance, including sensitivity, pointing accuracy, angular resolution, and so on. Several remarkable discoveries made in astrophysics associate with the Crab Nebula and its pulsar. One of the discoveries is that strong $\gamma$-ray flares with the energies above $100 \mathrm{MeV}$, which have been observed many times by AGILE[9] and FermiLAT[10] since 2007. The strongest emission, observed during the 2011 April "superflare", exceeded the quiescent level by more than several times. A $0.88 \mathrm{PeV} \gamma$-ray event from the Crab Nebula is recorded by the LHAASO detectors[11]. These observations challenge the standard models for particle acceleration in pulsar wind nebula. Several hypotheses have been put forward to explain this phenomenon, but the origin is still not fully understood, moreover UHE $\gamma$-ray flares have not been detected either.

LHAASO $\left(100.01^{\circ} \mathrm{E}, 29.35^{\circ} \mathrm{N}\right)$ is a large hybrid extensive air shower(EAS) array with an area of about $1 \mathrm{~km}^{2}$ at an altitude of $4410 \mathrm{~m}$ a.s.l. in Sichuan Province, China[12]. It consists of three sub-arrays: a $1.3 \mathrm{~km}^{2}$ array (KM2A) for $\gamma$-ray astronomy above $10 \mathrm{TeV}$ and cosmic ray physics, a $78000 \mathrm{~m}^{2}$ water Cherenkov detector array (WCDA) for $\mathrm{TeV} \gamma$-ray astronomy and a wide fieldof-view(FOV) air Cherenkov/fluorescence telescope array(WFCTA) for cosmic ray physics in the energy range from $10 \mathrm{TeV}$ to $1 \mathrm{EeV}$. The whole $\mathrm{KM} 2 \mathrm{~A}$ array is composed of 5195 electromagnetic detectors (EDs) and 1188 undersurface muon detectors (MDs), deployed over an area of $1.3 \mathrm{~km}^{2}$. KM2A has a wide FOV of 2 sr and covers $60 \%$ of the sky within a diurnal observation.

Half of the KM2A has been in operation with steady data-taking since December 2019. After a few months, the sensitivity for $\gamma$-ray sources at energies above $50 \mathrm{TeV}$ is already better than what has been achieved by previous observations[13]. At the end of 2020, the three quarters of KM2A started operation. The analysis results reported in this paper are based on the data collected from December 2019 to February 2021. For a more complete detailed description of the detector performance, $\mathrm{PeV} \gamma$-ray event, and the energy spectrum of the Crab Nebula, please see ref.[13, 14].

\section{Observations and analysis results}

\subsection{Data and MC sample}

We use CORSIKA program(version 7.6400)[15] to simulate the extensive air showers process initiated by primary particles in the atmosphere, and develop a software G4KM2A[16, 17] based on GEANT4(v4.10.00)[18] to obtain an accurate KM2A detector response. The Monte Carlo samples used in the present analysis are $2.222 \times 10^{8} \gamma$-ray shower and $4.444 \times 10^{8}$ proton shower events. Both 
the $\gamma$ ray and proton events were sampled in the energy range from $1 \mathrm{TeV}$ to $10 \mathrm{PeV}$ following a power-law function with a spectral index of -2 . The zenith angle is in the range from $0^{\circ}$ to $70^{\circ}$. The sample area is a circular region with a sufficiently large radius of $1000 \mathrm{~m}$.

\subsection{Detector performance}

The primary particle direction, energy and core location of the corresponding shower can be reconstructed by measuring the number of secondary particles and the arrival time. For KM2A events, only the ED hits with timing and charge information are used for direction, core location, and energy reconstruction. Both ED and MD hits are used for composition discrimination. Only hits with time residual within $[-30,50]$ ns perpendicular to the shower plane and with a distance less than $200 \mathrm{~m}$ from the shower axis are selected. The final surviving ED hits are used to count the number of electromagnetic particles(denoted as $N_{e}$ ). To reduce pollution from the punch-through high energy electromagnetic particles near the shower core, only MDs further than $15 \mathrm{~m}$ from the shower axis are used to obtain the number of muons $N_{\mu}$. The parameters $N_{\mu}$ and $N_{e}$ are used to discriminate between $\gamma$-ray showers and cosmic ray showers.

- Core and angular resolution

The shower core location is very important for direction reconstruction, which will use the core location as a vertex when fitting the shower front to a conical shape. An optimized centroid method is implemented to reconstruct the shower core position, and the functions are written as below :

$$
\text { Corex }=\frac{\sum w_{i} x_{i}}{\sum w_{i}}, \text { Corey }=\frac{\sum w_{i} y_{i}}{\sum w_{i}}, \text { Core } z=\frac{\sum w_{i} z_{i}}{\sum w_{i}}
$$

where $w_{i}=n_{e} e^{-\frac{1}{2}\left(r_{i} / 15\right)^{2}},\left(x_{i}, y_{i}, z_{i}\right)$ are the ED coordinates, $r_{i}$ is the ED distance to the shower core, the units are meter, and $n_{e}$ is the number of particles of ED. The obtained core location is used as an initial value for further core reconstruction. The lateral distribution of particle density measured by KM2A is fitted by the modified Nishimura-Kamata-Greisen(NKG)function[19]:

$$
\rho(r)=\frac{N_{\text {size }}}{2 \pi r_{m}^{2}} \frac{\Gamma(4.5-s)}{\Gamma(s-0.5) \Gamma(5-2 s)}\left(\frac{r}{r_{m}}\right)^{s-2.5}\left(1+\frac{r}{r_{m}}\right)^{s-4.5}
$$

where $r$ is the distance to the shower axis, $N_{\text {size }}$ is the total number of particles, $\mathrm{s}$ is the age of the shower, and $r_{m}$ is the Moliére radius. $r_{m}$ is fixed at $136 \mathrm{~m}$ [20]. The reconstruction parameters are the core location, $N_{\text {size }}$ and s. The core resolution is energy and zenith angle dependent. The core resolution for $\gamma$-ray events is shown in Fig.1a as a function of the reconstructed energy. The resolution (denoted as $R_{68}$, containing $68 \%$ of the events) is about $4 \sim 9 \mathrm{~m}$ at $20 \mathrm{TeV}$ and $2 \sim 4 \mathrm{~m}$ at $100 \mathrm{TeV}$.

The secondary particles of a shower travel roughly in a plane perpendicular to the direction of the primary particle, in fact, the shower front has a slightly conical shape, which should be considered. The shower direction can be obtained by minimizing the formula :

$$
\chi^{2}=\frac{1}{N_{h i t}} \sum_{i=1}^{N_{h i t}}\left[w_{i}\left(t_{i}-l \frac{x_{i}}{c}-m \frac{y_{i}}{c}-n \frac{z_{i}}{c}-\alpha \frac{r_{i}}{c}-t_{0}\right)\right]^{2}
$$

where $l=\sin \theta \cos \phi, m=\sin \theta \sin \phi, n=\cos \theta, \theta$ and $\phi$ are direction angles, $\alpha$ is the conical correction coefficient, and $c=0.2998 \mathrm{~m} / \mathrm{ns}$ is the speed of light. $t_{i}$ is the measured time of the $i$ th 


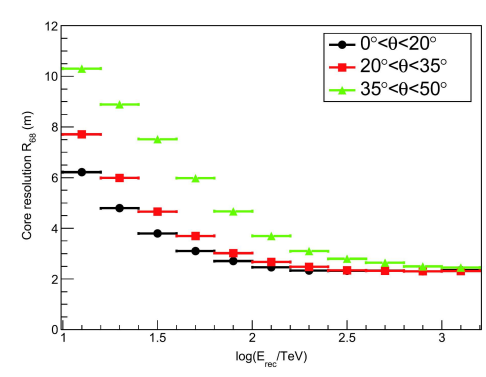

(a) Core resolution

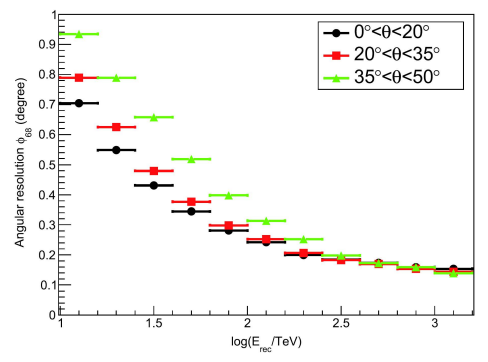

(b) Angular resolution

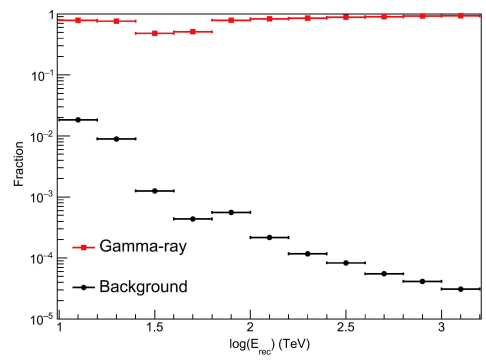

(c) Survival fraction of $\gamma$ ray

Figure 1: Core and angular resolution of the KM2A half array for simulated $\gamma$-ray showers. Survival fraction of $\gamma$ ray(MC) and cosmic ray background events(data) in different energy bins.

$\mathrm{ED},\left(x_{i}, y_{i}, z_{i}\right)$ are the $\mathrm{ED}$ coordinates, $r_{i}$ is the distance of ED location to the shower axis, and $w_{i}$ is a weight set according to the time residual and distance to the shower core. The angular resolution for $\gamma$-ray events is shown in Fig. $1 \mathrm{~b}$. The angular resolution (denoted as $\phi_{68}$, containing $68 \%$ of the events) is $0.5^{\circ} \sim 0.8^{\circ}$ at $20 \mathrm{TeV}$ and $0.24^{\circ} \sim 0.3^{\circ}$ at $100 \mathrm{TeV}$.

\section{- Background rejection power}

Most of the showers detected by KM2A are cosmic ray induced showers, which constitute the main background for $\gamma$-ray observations. Considering that $\gamma$ ray induced showers are muonpoor and cosmic ray induced showers are muon-rich, the ratio between the measured muons and electrons is used to discriminate the primary $\gamma$ rays from cosmic nuclei. The ratio is defined as : $R=\log \left(\frac{N_{\mu}+0.0001}{N_{e}}\right)$, where $N_{\mu}$ and $N_{e}$ are defined in the first paragraph of section2.2, and 0.0001 is used to show the cases with $N_{\mu}=0$. Fig.1c shows the survival fraction for $\gamma$-ray showers (from simulation) along with the measured survival fraction for the cosmic ray background (from observation data). The fraction for $\gamma$-ray showers varies from $48 \%$ to $93 \%$. The rejection power of cosmic ray induced showers is better than $4 \times 10^{3}$ at energies above $100 \mathrm{TeV}$.

\section{- Energy resolution}

The particle density at the optimal radius at which the uncertainty is minimized is a robust estimator of a shower energy in the ground-based experiments. For KM2A, the particle density at $r=50 \mathrm{~m}$ (denoted as $\rho_{50}$ ) evaluated by the Eq.(2) is used to estimate the $\gamma$-ray energy. The energy resolution values using densities from $\rho_{40}$ to $\rho_{70}$ are almost the same. As the atmospheric depth over which the shower develops is proportional to $\sec \theta$, the zenith angle effect has to be taken into account in the energy reconstruction. The final response formula between $\rho_{50}$ and the primary energy is given by $: \log \left(E_{\text {rec }} / \mathrm{TeV}\right)=a(\theta) \cdot\left(\log \left(\rho_{50}\right)\right)^{2}+b(\theta) \cdot \log \left(\rho_{50}\right)+c(\theta)$, where $a(\theta), b(\theta)$ and $c(\theta)$ are known constants, which are given as functions of $\sec \theta$. The energy resolution is energy and zenith angle dependent. Fig.2 shows the relation between the reconstructed energy $\left(E_{\text {rec }}\right)$ and the primary true energy $\left(E_{\text {true }}\right)$ over zenith angles $0^{\circ} \sim 50^{\circ}$. Events with reconstructed energy above $10 \mathrm{TeV}$ are divided into five bins per decade. The energy resolution for each energy bin over different zenith angles is shown in Fig2. For showers with zenith angle less than $20^{\circ}$, the resolution is about $24 \%$ at $20 \mathrm{TeV}$ and $13 \%$ at $100 \mathrm{TeV}$. 

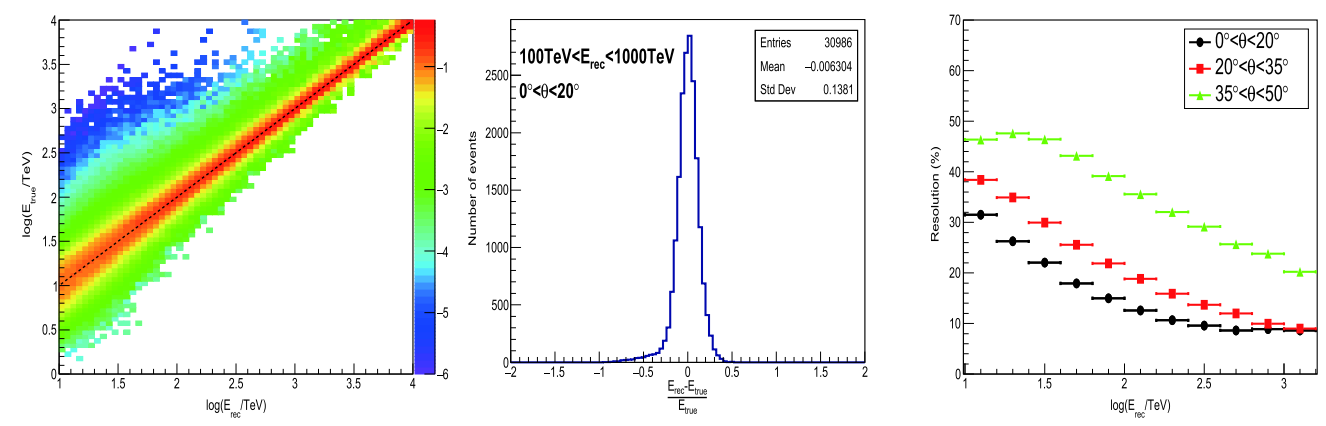

Figure 2: Left: event-by-event comparison of the primary true energy and the reconstructed energy for simulated $\gamma$-ray events over zenith angles $0^{\circ} \sim 50^{\circ}$. Middle: energy resolution function of showers in the energy range 100 1000 TeV with zenith angle $0^{\circ} \sim 20^{\circ}$. Right: dependence of energy resolution, defined as the half $68 \%$ width of the resolution function in each reconstructed energy bin.

\subsection{Energy spectrum}

On 11 January 2020 at 17:59:18 coordinated universal time (UTC), a giant air shower was recorded by all three LHAASO detectors(Fig.3). This event is verified as a $\gamma$ ray induced shower on the basis of 4996 particles (electrons, photons, muons, and hadrons) recorded by 395 surface detectors and 15 muons recorded by 10 under-surface detectors of KM2A. The chance probability for this event to be a misidentified CR is estimated as $0.1 \%$. Two independent estimates of the shower energy were derived from the KM2A and WFCTA data : $0.88 \pm 0.11 \mathrm{PeV}$ and $0.92{ }_{-0.20}^{+0.28}$ $\mathrm{PeV}$, respectively. The former value has previously been reported as the maximum energy of $\gamma$ ray detected from the Crab Nebula by LHAASO[11]. Approximately 1 year later, on 4 January 2021 at 16:45:06, another shower was registered by KM2A at even higher energy $(1.12 \pm 0.09 \mathrm{PeV})$. This event occurred at zenith angle $12.9^{\circ}$, closer to vertical and therefore better measured by KM2A than the previous shower. The number of detected secondary particles (5094) exceeded those in the previous event, the number of muons (14) was fewer, so we also identified this event as a $\gamma$ ray induced shower with a misidentification probability of $0.03 \%$.

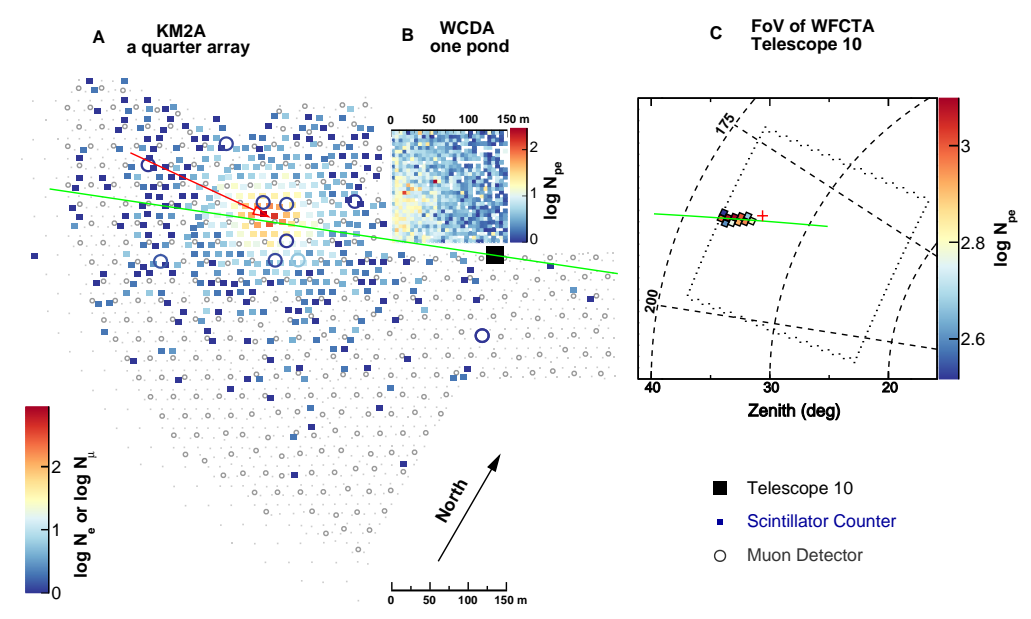

Figure 3: The $0.88 \mathrm{PeV} \gamma$-ray event from the Crab Nebula recorded by the LHAASO detectors. 
The $\gamma$-ray fluxes in the energy range from 0.5 to $13 \mathrm{TeV}$ were measured using the first pond of WCDA. From September 2019 to October 2020, the total exposure was 343.5 transits of the Crab Nebula. The KM2A measurement in the observation period covers the higher energy range from $10 \mathrm{TeV}$ to $1.1 \mathrm{PeV}$. We combined the WCDA and KM2A data to determine the SED of the Crab Nebula, The flux is shown in Fig.4a. The two independent measurements are consistent with a simple SED functional form, $d N / d E=(8.2 \pm 0.2) \times 10^{-14}(E / 10 T e V)^{-\Gamma} \mathrm{cm}^{-2} \mathrm{~s}^{-1} \mathrm{TeV}^{-1}$, where $\mathrm{N}$ is the number of $\gamma$ rays, $E$ is the $\gamma$-ray energy and $\Gamma$ is the energy dependent spectral index. The two measurements connect smoothly in the small overlapping region around $12.5 \mathrm{TeV}$. In this energy bin, the discrepancy between flux measured by KM2A and WCDA is $1.3 \sigma$. The functional form of the spectral index, $\Gamma=(2.90 \pm 0.01)+(0.19 \pm 0.02) \log _{10}(E / 10 \mathrm{TeV})$, implies a gradual steepening of the spectrum characterized by the local index $\Gamma$, from $\approx 2.5$ at $1 \mathrm{TeV}$ to 3.7 at $1 \mathrm{PeV}$. Fig. $4 \mathrm{a}$ also shows the result is consistent with previous measurements by other experiments in the low-energy region.

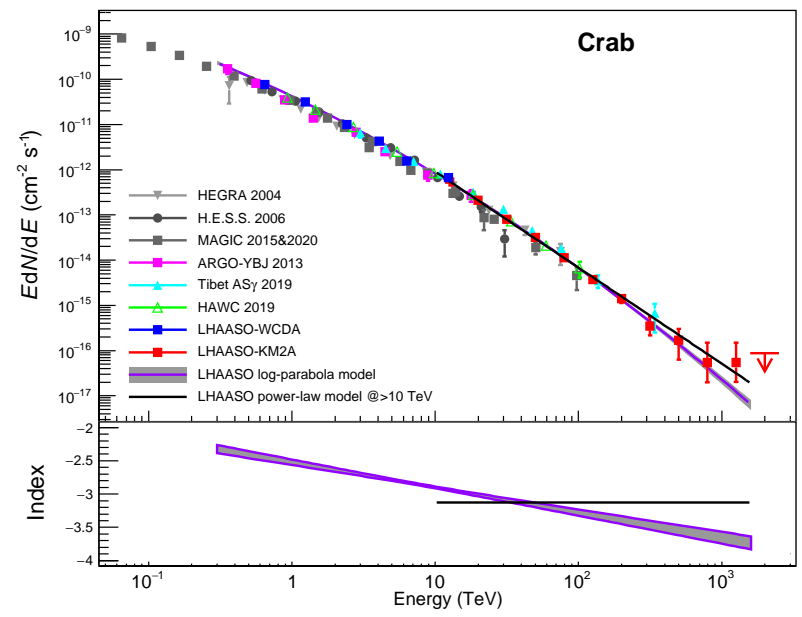

(a) Flux and fitting results

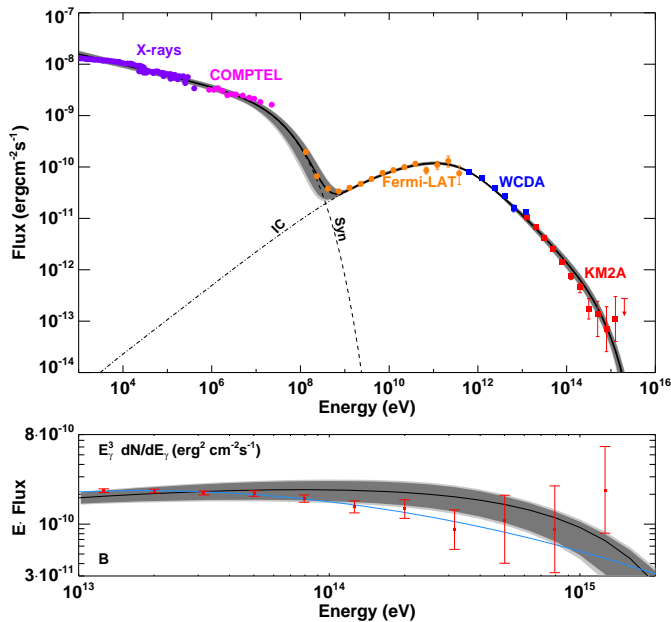

(b) Spectral energy distribution

Figure 4: Left : $\gamma$-ray flux of the Crab Nebula measured by LHAASO and spectral fitting. Right : The spectral energy distribution of the Crab Nebula.

The broad-band nonthermal emission of the Crab Nebula is dominated by two mechanisms synchrotron radiation and inverse Compton (IC) scattering of relativistic electrons interacting with the ambient magnetic and radiation fields[21, 22]. We modeled the Crab's multi wavelength radiation within the idealized synchrotron-IC one-zone model, assuming a homogeneous spatial distribution of the magnetic field and electrons (Fig.4b). For $E_{\gamma} \geq 100 \mathrm{TeV} \gamma$ rays, the dominant target for IC scattering is the $2.7 \mathrm{~K}$ cosmic microwave background radiation (CMBR), with properties that are known more precisely than the targets at lower energies. For a steady-state electron energy distribution, above $1 \mathrm{TeV}$, we assumed a power-law function terminated by a super-exponential cutoff at the high-energy end. Fig. $4 \mathrm{~b}$ shows that the SED model using three free parameters : the power-law slope $\alpha=3.42$, cutoff energy $E_{0}=2.15 \mathrm{PeV}$ and magnetic field $B=112 \mu \mathrm{G}$, which reproduces the observations from the $\mathrm{X}$-rays to multi-MeV $\gamma$ rays with synchrotron radiation and the $\mathrm{TeV}$ to $\mathrm{PeV} \gamma$ rays with IC radiation. Within the one-zone model, the IC $\gamma$-ray spectrum can be 
precisely calculated. Whereas the KM2A spectral points from $10 \mathrm{TeV}$ to $1 \mathrm{PeV}$ agree with the onezone model within the statistical uncertainties, there are possible deviations from its predictions. Between 60 and $500 \mathrm{TeV}$, the two differ with a significance of $4 \sigma$, with the observational data having a steeper spectrum than the one-zone model predictions. The possible excess around $1 \mathrm{PeV}$ might indicate an opposite tendency a hardening of the spectrum. A hardening of the electron spectrum is difficult to accommodate theoretically with plausible assumptions. The problem of suppression of the one-zone spectrum at $1 \mathrm{PeV}$ can be circumvented by introducing a second population of $\mathrm{PeV}$ electrons. This could also explain the inconsistency of the synchrotron part of the SED with the one-zone model by decoupling the highest energy synchrotron and IC components, assuming that the $\mathrm{MeV}$ synchrotron radiation is predominantly produced in compact, highly magnetized regions, whereas the PeV IC photons originate from regions with $B \leq 100 \mu \mathrm{G}$. A second electron component could extend the SED to a few PeV but not much further.

\subsection{Search for $\gamma$-ray flares}

The "Equi-Zenith angle Method" is adopted to estimate the number of cosmic ray background events from the Crab Nebula, and the on-source live time of observation of the Crab Nebula is corrected. No UHE flares on the timescale of days are observed with a rough search. We will optimize the flares monitor software for high sensitivity to the light variation and keep monitoring the Crab Nebula.

\section{Summary}

Half of the KM2A detector performance has been verified, including angular resolution, core resolution, $\gamma$ ray/background discrimination, and energy resolution, and the results show half of the KM2A has an excellent performance. The energy spectrum of the Crab Nebula is measured, and the energy range is extended to $1.1 \mathrm{PeV}$. The energy dependent spectral index implies a gradual steepening from 2.5 at $1 \mathrm{TeV}$ to 3.7 at $1 \mathrm{PeV}$. Within the idealized Synchrotron-IC one-zone model, the KM2A spectral points from $10 \mathrm{TeV}$ to $1 \mathrm{PeV}$ agree with the IC $\gamma$-ray prediction within the statistical uncertainties. Between 60 and $500 \mathrm{TeV}$, a deviation of $4 \sigma$ significance indicates a steeper spectrum than the one-zone model predictions. Whether leptonic or hadronic origin of the $\gamma$-ray emission above $60 \mathrm{TeV}$ can not be confirmed with the inadequate statistics at present.

\section{References}

[1] D. A. Green 2003, in Supernovae and $\gamma$-ray Bursters, ed. K. Weiler, Lect. Notes Phys. (Berlin: Springer Verlag), 598, 7

[2] B. Bartoli, P. Bernardini, X. J. Bi et al., ApJ 779, 27 (2013)

[3] B. Bartoli, P. Bernardini, X. J. Bi et al., ApJ 798, 119(2015)

[4] A. U. Abeysekara, A. Albert, R. Alfaro et al., ApJ 881, 134(2019)

[5] M. Amenomori et al., PRL 123, 051101(2019) 
[6] F. A. Aharonian et al., ApJ 614, 897(2004)

[7] F. Aharonian et al., A\&A 457, 899(2006)

[8] J. Aleksić et al., Journal of High Energy Astrophysics 5-6, 30-38(2015)

[9] Tavani M. et al. Science, 2011, 331: 736-739

[10] A. A. Abdo et al. Science, 2011, 331: 739-742

[11] Cao, Z., Aharonian, F.A., An, Q. et al. Nature, 2021, 594: 33-36

[12] H. H. He, for the LHAASO Collaboration, RDTM, 2018, 2:7

[13] F. Aharonian et al., 2021 Chinese Phys. C 45025002

[14] The LHAASO Collaboration, Science 10.1126/science.abg5137 (2021).

[15] D. Heck, et al., 1998, Forschungszentrum Karlsruhe Report No. FZKA 6019

[16] S. Z. Chen et al., Nuclear Electronics \& Detection Technology 37, 1101-1105 (2017)

[17] S. Z. Chen et al., proceeding of 36th ICRC, (2019)

[18] S. Agostinelli,et al., NIMA 506, 250 (2003)

[19] K. Greisen, Ann. Rev. Nucl. Part. Sci. 10, 63 (1960)

[20] K. F. Grieder Peter, Extensive Air Showers High Energy Phenomena and Astrophysical Aspects, Springer-Verlag Berlin Heidelberg 1, 193 (2010)

[21] O. C. de Jager, A. K. Harding, Astrophys. J. 396. 161(1992)

[22] A. M. Atoyan, F. A. Aharonian, Mon. Not. R. Astron. Soc. 278, 525(1996) 


\section{Full Authors List: LHAASO Collaboration}

Zhen $\mathrm{Cao}^{1,2,3}$, F. Aharonian ${ }^{4,5}$, Q. An ${ }^{6,7}$, Axikegu $^{8}$, L.X. Bai ${ }^{9}$, Y.X. Bai ${ }^{1,3}$, L.X. Bai ${ }^{9}$, Y.X. Bai ${ }^{1,3}$, Y.W. Bao ${ }^{10}$, D. Bastieri ${ }^{11}$, X.J. Bi ${ }^{1,2,3}$, Y.J. Bi ${ }^{1,3}$, H. Cai ${ }^{12}$, J.T. Cai ${ }^{11}$, Zhe Cao ${ }^{6,7}$, J. Chang ${ }^{13}$, J.F. Chang ${ }^{1,3,6}$, B.M. Chen ${ }^{14}$, E.S. Chen ${ }^{1,2,3}$, J. Chen ${ }^{9}$, Liang Chen $^{1,2,3}$, Liang Chen ${ }^{15}$, Long Chen ${ }^{8}$, M.J. Chen ${ }^{1,3}$, M.L. Chen ${ }^{1,3,6}$, Q.H. Chen ${ }^{8}$, S.H. Chen ${ }^{1,2,3}$, S.Z. Chen ${ }^{1,3}$, T.L. Chen ${ }^{16}$,X.L. $\mathrm{Chen}^{1,2,3}$, Y. Chen $^{10}$, N. Cheng ${ }^{1,3}$, Y.D. Cheng ${ }^{1,3}$, S.W. Cui ${ }^{14}$, X.H. Cuii ${ }^{17}$, Y.D. Cui ${ }^{18}$, B. D'Ettorre Piazzoli ${ }^{19}$, B.Z. Dai ${ }^{20}$, H.L. Dai $^{1,3,6}$, Z.G. Dai ${ }^{7}$, Danzengluobu ${ }^{16}$, D. della Volpe ${ }^{21}$, X.J. Dong ${ }^{1,3}$, K.K. Duan ${ }^{13}$, J.H. Fan ${ }^{11}$, Y.Z. Fan ${ }^{13}$, Z.X. Fan ${ }^{1,3}$, J. Fang ${ }^{20}$, K. Fang $^{1,3}$, C.F. Feng ${ }^{22}$, L. Feng ${ }^{13}$, S.H. Feng ${ }^{1,3}$, Y.L. Feng ${ }^{13}$, B. Gao ${ }^{1,3}$, C.D. Gao ${ }^{22}$, L.Q. Gao ${ }^{1,2,3}$, Q. Gao ${ }^{16}$, W. Gao ${ }^{22}$, M.M. Ge $^{20}$, L.S. Geng ${ }^{1,3}$, G.H. Gong ${ }^{23}$, Q.B. Gou ${ }^{1,3}$, M.H. Gu${ }^{1,3,6}$, F.L. Guo ${ }^{15}$, J.G. Guo ${ }^{1,2,3}$, X.L. Guo ${ }^{8}$, Y.Q. Guo ${ }^{1,3}$, Y.Y. Guo ${ }^{1,2,3,13}$, Y.A. $\mathrm{Han}^{24}$, H.H. He $\mathrm{H}^{1,2,3}$, H.N. He ${ }^{13}$, J.C. He ${ }^{1,2,3}$, S.L. He ${ }^{11}$, X.B. He ${ }^{18}$, Y. He ${ }^{8}$, M. Heller ${ }^{21}$, Y.K. Hor ${ }^{18}$, C. Hou ${ }^{1,3}$, H.B. Hu ${ }^{1,2,3}$, S.

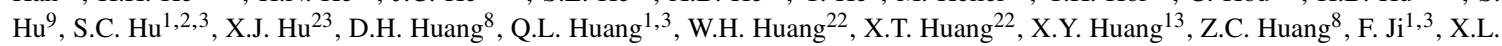
$\mathrm{Ji}^{1,3,6}$, H.Y. Jia ${ }^{8}$, K. Jiang ${ }^{6,7}$, Z.J. Jiang ${ }^{20}$, C. Jin ${ }^{1,2,3}$, T. Ke ${ }^{1,3}$, D. Kuleshov ${ }^{25}$, K. Levochkin ${ }^{25}$, B.B. Li ${ }^{14}$, Cheng Li ${ }^{6,7}$, Cong Li ${ }^{1,3}$,

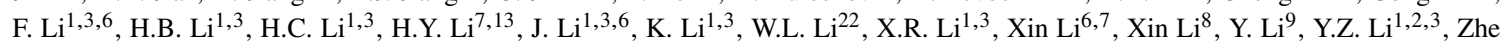

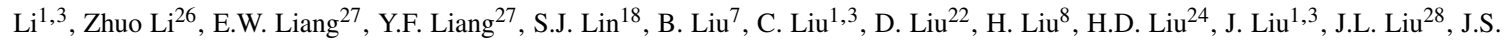

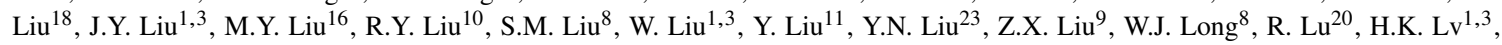
B.Q. $\mathrm{Ma}^{26}$, L.L. $\mathrm{Ma}^{1,3}$, X.H. Ma ${ }^{1,3}$, J.R. $\mathrm{Mao}^{29}$, A. $\operatorname{Masood}^{8}$, Z. $\mathrm{Min}^{1,3}$, W. Mitthumsiri' ${ }^{30}$, T. Montaruli ${ }^{21}$, Y.C. Nan ${ }^{22}$, B.Y. Pang ${ }^{8}$, P. Pattarakijwanich ${ }^{30}$, Z.Y. Pei ${ }^{11}$, M.Y. Qi ${ }^{1,3}$, Y.Q. Qi ${ }^{14}$, B.Q. Qiao ${ }^{1,3}$, J.J. Qin ${ }^{7}$, D. Ruffolo ${ }^{30}$, V. Rulev ${ }^{25}$, A. Sáiz ${ }^{30}$, L. Shao ${ }^{14}$, O. Shchegolev $^{25,31}$, X.D. Sheng ${ }^{1,3}$, J.Y. Shi ${ }^{1,3}$, H.C. Song ${ }^{26}$, Yu.V. Stenkin ${ }^{25,31}$, V. Stepanov ${ }^{25}$, Y. Su ${ }^{32}$, Q.N. Sun ${ }^{8}$, X.N. Sun ${ }^{27}$, Z.B.

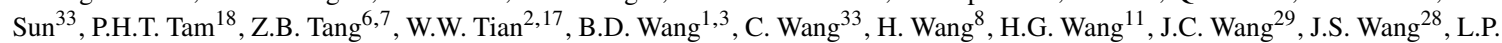
Wang $^{22}$, L.Y. Wang ${ }^{1,3}$, R.N. Wang ${ }^{8}$, W. Wang ${ }^{18}$, W. Wang ${ }^{12}$, X.G. Wang ${ }^{27}$, X.J. Wang ${ }^{1,3}$, X.Y. Wang ${ }^{10}$, Y. Wang ${ }^{8}$, Y.D. Wang ${ }^{1,3}$, Y.J. Wang $^{1,3}$, Y.P. Wang ${ }^{1,2,3}$, Z.H. Wang ${ }^{9}$, Z.X. Wang ${ }^{20}$, Zhen Wang ${ }^{28}$, Zheng Wang ${ }^{1,3,6}$, D.M. Wei $^{13}$, J.J. Wei ${ }^{13}$, Y.J. Wei ${ }^{1,2,3}$, T. Wen ${ }^{20}$, C.Y. $\mathrm{Wu}^{1,3}$, H.R. $\mathrm{Wu}^{1,3}$, S. Wu ${ }^{1,3}$, W.X. Wu ${ }^{8}$, X.F. Wu ${ }^{13}$, S.Q. Xi ${ }^{1,3}$, J. Xia ${ }^{7,13}$, J.J. Xia ${ }^{8}$, G.M. Xiang ${ }^{2,15}$, D.X. Xiao ${ }^{16}$, G. Xiao ${ }^{1,3}$, H.B. Xiao ${ }^{11}$, G.G. Xin ${ }^{12}$, Y.L. Xin ${ }^{8}$, Y. Xing ${ }^{15}$, D.L. Xu ${ }^{28}$, R.X. Xu ${ }^{26}$, L. Xue ${ }^{22}$, D.H. Yan ${ }^{29}$, J.Z. Yan ${ }^{13}$, C.W. Yang ${ }^{9}$, F.F. Yang ${ }^{1,3,6}$, J.Y. Yang ${ }^{18}$, L.L. Yang ${ }^{18}$, M.J. Yang ${ }^{1,3}$, R.Z. Yang ${ }^{7}$, S.B. Yang ${ }^{20}$, Y.H. Yao ${ }^{9}$, Z.G. Yao ${ }^{1,3}$, Y.M. Ye ${ }^{23}$, L.Q. Yin ${ }^{1,3}$, N. Yin ${ }^{22}$, X.H. You $^{1,3}$, Z.Y. You ${ }^{1,2,3}$, Y.H. Yu ${ }^{22}$, Q. Yuan ${ }^{13}$, H.D. Zeng ${ }^{13}$, T.X. Zeng ${ }^{1,3,6}$, W. Zeng ${ }^{20}$, Z.K. Zeng ${ }^{1,2,3}$, M. Zha ${ }^{1,3}$, X.X. Zhai ${ }^{1,3}$, B.B. Zhang ${ }^{10}$, H.M. Zhang ${ }^{10}$, H.Y. Zhang ${ }^{22}$, J.L. Zhang ${ }^{17}$, J.W. Zhang ${ }^{9}$, L.X. Zhang ${ }^{11}$, Li Zhang ${ }^{20}$, Lu Zhang ${ }^{14}$, P.F. Zhang ${ }^{20}$, P.P. Zhang ${ }^{14}$, R. Zhang ${ }^{7,13}$, S.R. Zhang ${ }^{14}$, S.S. Zhang ${ }^{1,3}$, X. Zhang ${ }^{10}$, X.P. Zhang ${ }^{1,3}$, Y.F. Zhang ${ }^{8}$, Y.L. Zhang ${ }^{1,3}$, Yi Zhang ${ }^{1,13}$, Yong Zhang ${ }^{1,3}$, B. Zhao $^{8}$, J. Zhao ${ }^{1,3}$, L. Zhao ${ }^{6,7}$, L.Z. Zhao ${ }^{14}$, S.P. Zhao ${ }^{13,22}$, F. Zheng ${ }^{33}$, Y. Zheng ${ }^{8}$, B. Zhou ${ }^{1,3}$, H. Zhou ${ }^{28}$, J.N. Zhou ${ }^{15}$, P. Zhou ${ }^{10}$, R. Zhou $^{9}$, X.X. Zhou ${ }^{8}$, C.G. Zhu ${ }^{22}$, F.R. Zhu ${ }^{8}$, H. Zhu ${ }^{17}$, K.J. Zhu ${ }^{1,2,3,6}$ and X. Zuo ${ }^{1,3}$

${ }^{1}$ Key Laboratory of Particle Astrophyics \& Experimental Physics Division \& Computing Center, Institute of High Energy Physics, Chinese Academy of Sciences, 100049 Beijing, China.

${ }^{2}$ University of Chinese Academy of Sciences, 100049 Beijing, China.

${ }^{3}$ TIANFU Cosmic Ray Research Center, Chengdu, Sichuan, China.

${ }^{4}$ Dublin Institute for Advanced Studies, 31 Fitzwilliam Place, 2 Dublin, Ireland.

${ }^{5}$ Max-Planck-Institut for Nuclear Physics, P.O. Box 103980, 69029 Heidelberg, Germany.

${ }^{6}$ State Key Laboratory of Particle Detection and Electronics, China.

${ }^{7}$ University of Science and Technology of China, 230026 Hefei, Anhui, China.

${ }^{8}$ School of Physical Science and Technology \& School of Information Science and Technology, Southwest Jiaotong University, 610031 Chengdu, Sichuan, China.

${ }^{9}$ College of Physics, Sichuan University, 610065 Chengdu, Sichuan, China

${ }^{10}$ School of Astronomy and Space Science, Nanjing University, 210023 Nanjing, Jiangsu, China.

${ }^{11}$ Center for Astrophysics, Guangzhou University, 510006 Guangzhou, Guangdong, China.

${ }^{12}$ School of Physics and Technology, Wuhan University, 430072 Wuhan, Hubei, China.

${ }^{13}$ Key Laboratory of Dark Matter and Space Astronomy, Purple Mountain Observatory, Chinese Academy of Sciences, 210023 Nanjing, Jiangsu, China.

${ }^{14}$ Hebei Normal University, 050024 Shijiazhuang, Hebei, China.

${ }^{15}$ Key Laboratory for Research in Galaxies and Cosmology, Shanghai Astronomical Observatory, Chinese Academy of Sciences, 200030 Shanghai, China.

${ }^{16}$ Key Laboratory of Cosmic Rays (Tibet University), Ministry of Education, 850000 Lhasa, Tibet, China.

${ }^{17}$ National Astronomical Observatories, Chinese Academy of Sciences, 100101 Beijing, China.

${ }^{18}$ School of Physics and Astronomy \& School of Physics (Guangzhou), Sun Yat-sen University, 519000 Zhuhai, Guangdong, China.

${ }^{19}$ Dipartimento di Fisica dell'Università di Napoli 'Federico II”, Complesso Universitario di Monte Sant’Angelo, via Cinthia, 80126 Napoli, Italy.

${ }^{20}$ School of Physics and Astronomy, Yunnan University, 650091 Kunming, Yunnan, China.

${ }^{21}$ Dąŕepartement de Physique Nuclą́reaire et Corpusculaire, Facultąre de Sciences, Universitąŕe de Gen 'eve, 24 Quai Ernest Ansermet, 1211 Geneva, Switzerland.

${ }^{22}$ Institute of Frontier and Interdisciplinary Science, Shandong University, 266237 Qingdao, Shandong, China.

${ }^{23}$ Department of Engineering Physics, Tsinghua University, 100084 Beijing, China.

${ }^{24}$ School of Physics and Microelectronics, Zhengzhou University, 450001 Zhengzhou, Henan, China.

${ }^{25}$ Institute for Nuclear Research of Russian Academy of Sciences, 117312 Moscow, Russia. 
${ }^{26}$ School of Physics, Peking University, 100871 Beijing, China.

${ }^{27}$ School of Physical Science and Technology, Guangxi University, 530004 Nanning, Guangxi, China.

${ }^{28}$ Tsung-Dao Lee Institute \& School of Physics and Astronomy, Shanghai Jiao Tong University, 200240 Shanghai, China.

${ }^{29}$ Yunnan Observatories, Chinese Academy of Sciences, 650216 Kunming, Yunnan, China.

${ }^{30}$ Department of Physics, Faculty of Science, Mahidol University, 10400 Bangkok, Thailand.

${ }^{31}$ Moscow Institute of Physics and Technology, 141700 Moscow, Russia.

${ }^{32}$ Key Laboratory of Radio Astronomy, Purple Mountain Observatory, Chinese Academy of Sciences, 210023 Nanjing, Jiangsu, China.

${ }^{33}$ National Space Science Center, Chinese Academy of Sciences, 100190 Beijing, China. 